Ryner Jose D. Carrillo, MD, MSc ${ }^{1,2}$

Jose Florencio F. Lapeña Jr, MA, MD²

'Department of Anatomy, College of Medicine University of the Philippines Manila

2Department of Otorhinolaryngology, College of Medicine - Philippine General Hospital University of the Philippines Manila
Correspondence: Dr. Ryner Jose D. Carrillo Department of Anatomy, UP College of Medicine University of the Philippines Manila

Pedro Gil St., Ermita, Manila 1000

Phone: (632) 5264194

Email: rynercarrillo@gmail.com

The authors declared that this represents original material, that the manuscript has been read and approved by the authors, that the requirements for authorship have been met by each author, and that the authors believe that the manuscript represents honest work

This research was registered with the University of the Philippines Manila (UPM) Research Grants Administration Office (RGAO-2018-0963) and UPM Research Ethics Board exemption was obtained to report this series.

Disclosures: The authors signed disclosures that there are no financial or other (including personal) relationships, intellectual passion, political or religious beliefs, and institutional affiliations that might lead to a conflict of interest.

\section{Can Modified Laryngosternopexy (Laryngoclaviculopexy) Project the Larynx Anteriorly?}

Laryngosternopexy is a suturing method between the thyroid lamina and sternal ligament in order to relieve tension from the anastomosis when performing segmental resection of the airway. A thick absorbable monofilament suture is passed through thyroid lamina and the interclavicular ligament of the sternum in a figure of eight fashion as described by Castellanos. ${ }^{1,2}$ In laryngosternopexy, the suture support is ventral to the anastomotic site in the midline. However, this places the "pexy" sutures in the way, making a tracheotomy and second stage decannulation difficult. We describe a modified laryngosternopexy (laryngoclaviculopexy) that can be performed with the "pexy" sutures out of the way to allow access to the trachea and our initial experience with three patients.

\section{METHODS}

This modification of suturing utilizes the anterior sternoclavicular ligaments bilaterally instead of the interclavicular ligaments overlying the manubrium. (Figure 1 A, B) After completing the cricotracheal resection or laryngotracheal - anastomosis in the usual manner, the thick absorbable monofilament suture such as polydioxanone PDS II 2-0 (Ethicon, NJ, USA) is passed through the ipsilateral thyroid lamina and sternoclavicular ligaments bilaterally, deeply enough to avoid cheese-wiring.

This is illustrated in representative photos of modified laryngosternopexy (laryngoclaviculopexy) following cricotracheal resection (Figure 2A) and laryngotracheal resection - anastomosis (Figure 2B). Theoretically, the anterior triangle orientation of the laryngosternopexy should provide a stable support anteriorly and laterally especially in accidental hyperextension and side-to-side turning of the neck. The question is, can this laryngoclaviculopexy bring down the laryngeal framework to project it anteriorly (ventrally)? 


\section{SURGICAL INNOVATIONS AND INSTRUMENTATION}

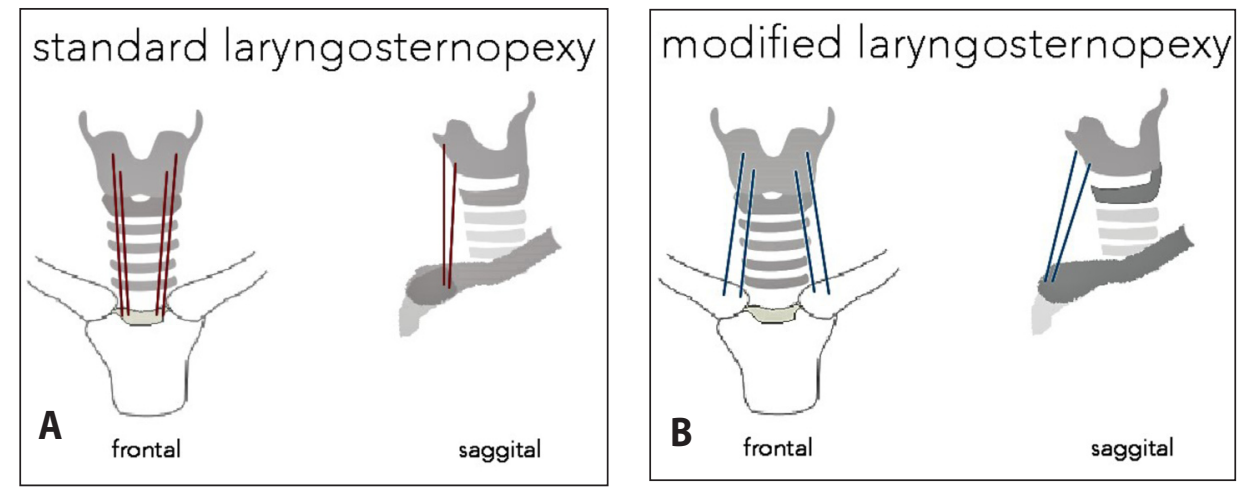

Figure 1. A. Standard laryngosternopexy with tension sutures directed along the direction of the trachea. B. Modified laryngosternopexy (laryngoclaviculopexy) with tension sutures directed anterolaterally to the sternoclavicular joints.
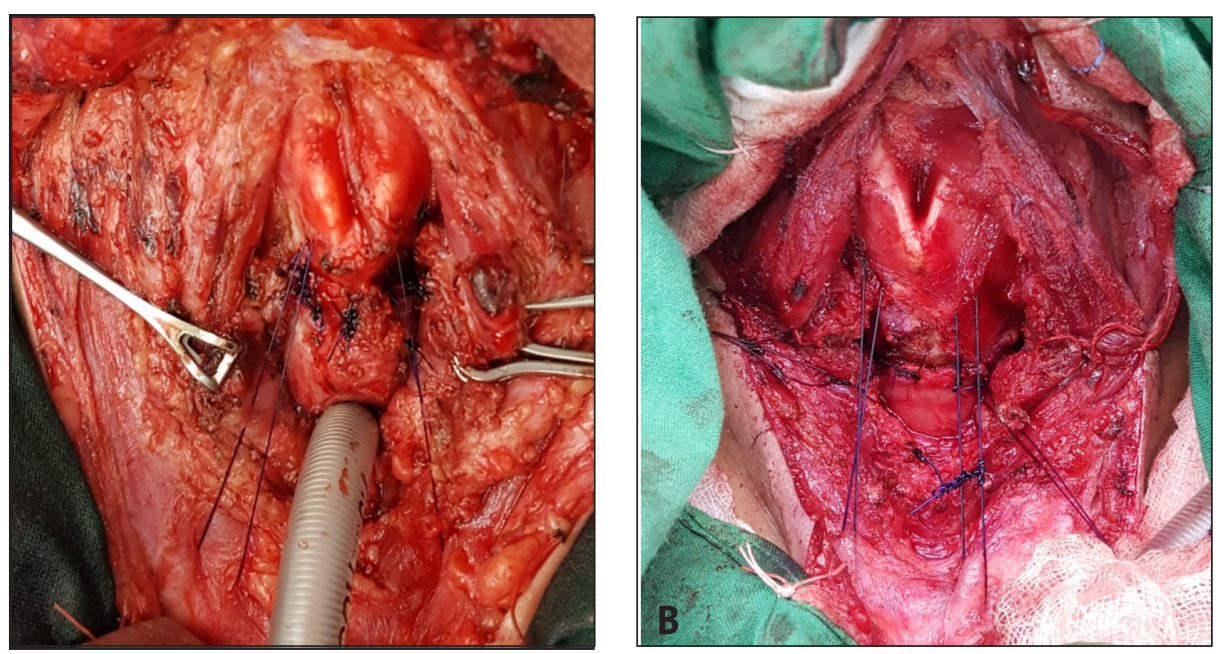

Figure 2. A. Cricotracheal resection and modified laryngosternopexy with tracheotomy for a second stage decannulation. B. Tracheal resection and modified laryngosternopexy provide adequate middle space should there be a need for tracheostomy.

\section{RESULTS}

With informed consent, modified laryngosternopexy was performed in three airway stenosis reconstructions. The cases were a partial cricotracheal resection and two segmental tracheal resectionanastamoses.

Case 1. An 18-year-old man sustained multiple injuries due to a vehicular crash. He underwent craniectomy and subsequent tracheotomy but developed subglottic stenosis involving cricoid and tracheal rings. Laser dilatation was not successful and he subsequently underwent partial cricotracheal resection maintaining a posterior cricoid shell to allow intussuception of the distal trachea and suturing directly to the thyroid framework. A modified laryngosternopexy and a tracheotomy for a grade IV subglottic stenosis were performed. The sternoclavicular ligaments were sufficiently robust and the "pexy" sutures did not cut out. The resected airway measured $4.5 \mathrm{~cm}$. He was successfully decannulated six months later and a CT scan of the airway showed normal laryngeal framework anterior projection. (Figure 3)
Case 2. A 28-year-old man suffered a motorcycle accident resulting in multiple injuries and subsequent repeated intubations and extubations. He developed grade IV subglottic stenosis after two weeks for which he was tracheotomized. Surgical airway evaluation showed tracheal stenosis. He underwent resection of $4.5 \mathrm{~cm}$ trachea, cricotracheal anastomosis without tracheotomy and modified laryngosternospexy to facilitate a possible emergency tracheotomy in the event of dehiscence. The toughness of the sternoclavicular ligaments was easily determined during the suturing process, and the tension "pexy" sutures were sufficiently placed to avoid cheesewiring. A CT scan of the airway after 2 months showed normal laryngeal framework anterior projection with only mild webbing at the cricotracheal anastomotic site. (Figure 4)

Case 3. A 51-year-old man sustained electrical injuries at work, resulting in multiple burns and subsequent prolonged intubation. He developed tracheal stenosis and subsequently underwent a tracheotomy with multiple unsuccessful attempts at decannulation. He was referred to us and a $4.5 \mathrm{~cm}$ tracheal resection with cricotracheal 


\section{SURGICAL INNOVATIONS AND INSTRUMENTATION}

anastomosis was performed. The modified laryngosternopexy (laryngoclaviculopexy) was easily achieved with sutures firmly embedded in the sternoclavicular ligaments. He was successfully extubated after 24 hours and discharged uneventfully. A CT scan 1.5 years post-surgery showed good patency and normal anterior projection of the thyroid cartilage framework. (Figure 5) hypothetically adequate stability provided should the patient suddenly turn the head to the right or left. The triangular configuration provides lateral traction to prevent sideways movement of the laryngeal framework away from the anastomotic site. We did not experience any complications related to instability in any of our 3 patients and had satisfactory follow up courses documented by CT scans. None of

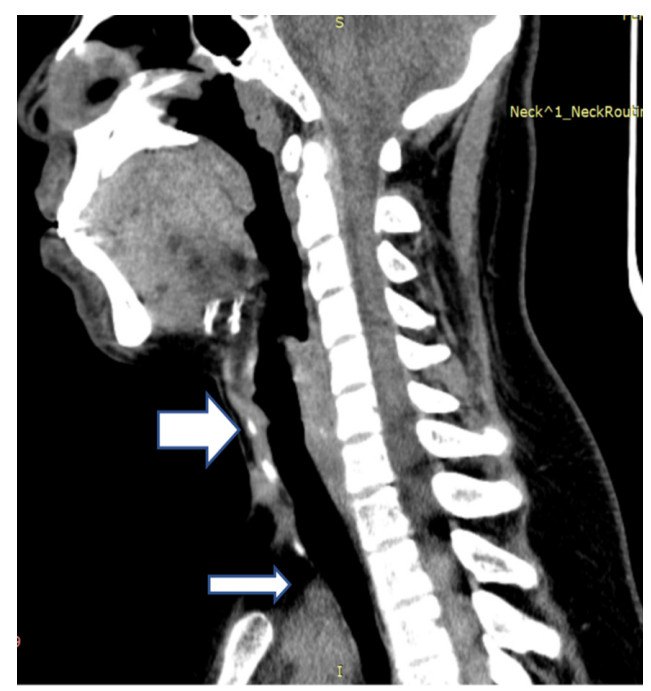

Figure 3. Partial cricotracheal resection with modified laryngosternopexy and a tracheostomy showing anterior and lateral traction of thyroid cartilage towards the sternoclavicular ligaments. Six months post decannulation, CT scan showed normal laryngeal
framework projection and patent airway (big arrow). Thinned skin from distal tracheotomy site is noticeable (small arrow).

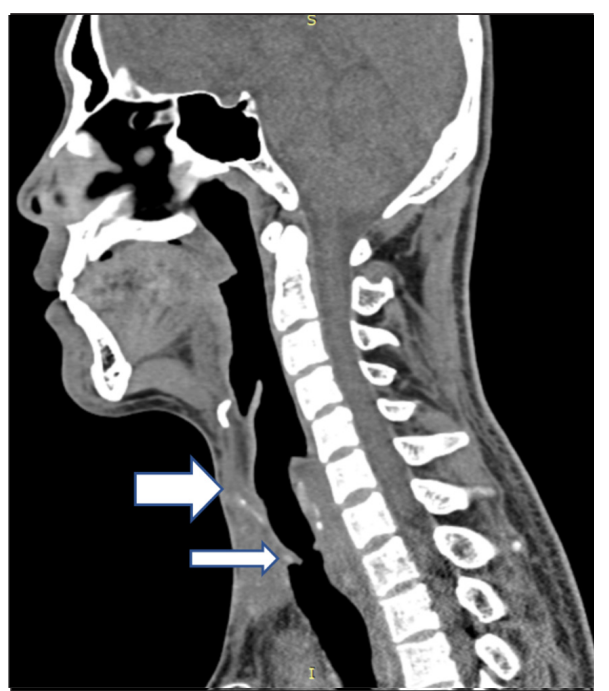

Figure 4. Tracheal resection with cricotracheal anastomosis and modified laryngosternopexy is shown. A CT scan 2 month after tracheal resection showing normal laryngeal framework projection (big arrow). Noticeable is a mild webbing at the level
of cricotracheal anastomotic site (small arrow), that is being closely monitored, although the patient is asymptomatic.

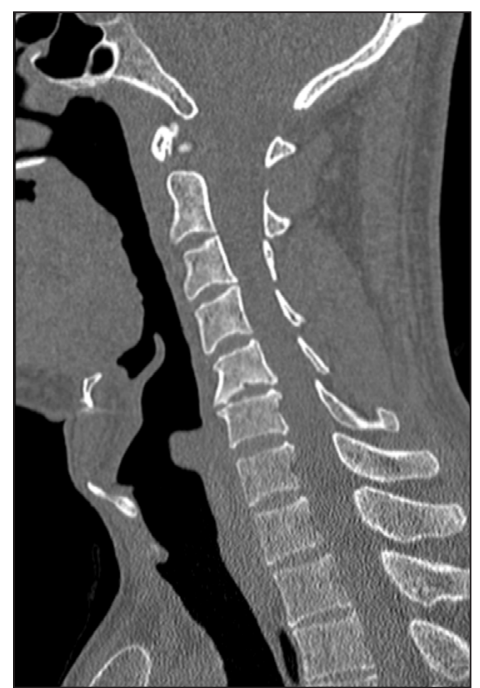

Figure 5. CT scan post tracheal resection with cricotracheal anastomosis and modified laryngosternopexy after 1.5 years. The patient comfortably performs activities of daily living and there are no signs of restenosis.

\section{DISCUSSION}

This preliminary series demonstrates that modified laryngosternopexy (laryngoclaviculopexy) brings down the laryngeal framework sufficiently to project it anteriorly. It may provide sufficient anterior and lateral traction forces between the thyroid cartilage and the sternoclavicular joints, as ligaments and muscles suspending the larynx superiorly and posteriorly from the hyoid prevent anterior rotation of the laryngeal framework.

Unlike the Grillo stitch ${ }^{4}$ which projects the head anteriorly in order to shorten the neck and lessen traction on the airway anastomosis, the standard laryngosternopexy provides traction directly between proximal and distal airway segments. However, options for second stage decannulation after reconstruction or for emergent airway reestablishment via tracheotomy are not easy with laryngosternopexy sutures in the way. Modifying this by utilizing the anterolateral sternoclavicular ligaments instead of the anteromedial interclavicular ligament provides space for elective or emergency tracheostomy with the "pexy" sutures out of the way. The sternoclavicular ligaments are robust enough to maintain the tension sutures and the modification does not tilt the laryngeal framework significantly. Moreover, there is our patients reported dysphagia, and no formal laryngeal release was performed in any of our patients.

Our initial experience suggests that modified laryngosternopexy (laryngoclaviculopexy) may be a practical and effective technique in airway reconstruction that maintains laryngeal airway projection while providing adequate space for second stage decannulation or repeat emergent tracheotomy. Formal trials may further prove our experience.

\footnotetext{
ACKNOWLEDGEMENTS

We thank Jose Pedrito M. Magno, MD for the line drawings in Figure 1.

\section{REFERENCES}

1. Castellanos PF. Laryngosternopexy [Youtube Video 2011 May 14; cited 2018 Apr 5]. Available from https://www.youtube.com/watch?v=23Kweq21z8k.

2. Castellanos PF. LSP surgery photos. [cited 2018 Apr 5] Available from: http://airwaysurgeon.com/ AirwaySurgeon/LSP_Surgery_Figures.html.

3. Atallah I, Aldkhyval A, Castellanos PF. Modified single-stage segmental cricotracheal resection. Eur Arch Otorhinolarygol. 2018 Jan;275(1): 139-146. DOI: 10.1007/s00405-017-4753-y; PMID: 29063231.

4. Kotake Y, Grillo HC. Reduction of tension at the anastomosis following tracheal resection in puppies. J Thoracic Cardiovasc Surg. 1976 Apr. 71(4):600-4.
} 